\section{Combinatorial Approaches to the Synthesis of Vapor Detector Arrays for Use in an Electronic Nose}

Adam J. Matzger, Carolyn E. Lawrence, Robert H. Grubbs, * and Nathan S. Lewis*

Division of Chemistry and Chemical Engineering, California Institute of Technology, Pasadena, California 91125

Received October 4, 1999

\section{Introduction}

The technology of "electronic noses", i.e., devices that functionally mimic the sense of olfaction, is rapidly evolving, driven by the practical demand for objective analysis of odors as well as by the intellectual challenge of mimicking the mammalian sense of olfaction. ${ }^{1-3}$ Numerous implementations of artificial noses have emerged; however, the most prevalent devices are based on detecting a physical or chemical change in a polymer film upon exposure to a gaseous analyte. ${ }^{4}$ The measured quantity in an individual detector can be the frequency shift of a resonating crystal in a quartz crystal microbalance $(\mathrm{QCM})^{5}$ or a surface acoustic wave configuration (SAW), ${ }^{6,7}$ changes in the optical absorption or emission properties of a dye that has been impregnated into a polymer, ${ }^{8-10}$ or changes in the electrical resistance of a conductive polymer $(\mathrm{cp})^{11}$ or of a carbon black/polymer composite (cb/pc) film. ${ }^{12}$ Any individual detector does not respond highly specifically toward an individual analyte, but the pattern of responses in an array of differentially responsive detectors can be used to identify, classify, and in some cases quantify, the analyte of interest. The common threads that tie these methods together are (a) their reliance on sorption of an analyte into the polymer film to produce the physicochemical effect that leads to the signal transduction event in the detectors of the array, and (b) the use of pattern recognition to send the collection of signals to a central processing unit for analysis.

Like the artificial olfactory systems, there is mounting evidence that mammals do not employ lock-and-key type specificity to individual analytes in the broadly responsive portion of their olfactory systems. ${ }^{13}$ Instead, humans, mice, and other mammals are believed to rely on a pattern generated from the response of many broadly tuned olfactory receptor sites, just as the individual polymer types in an electronic nose are swollen by many chemically diverse analytes. Humans are thought to have $\sim 1000$ different olfactory genes that presumably encode for $\sim 1000$ olfactory receptor proteins. A major goal of developing a functional mimic for the mammalian olfactory system is therefore to produce a highly diverse array of differentially responsive vapor detectors.

* Authors to whom correspondence should be addressed.
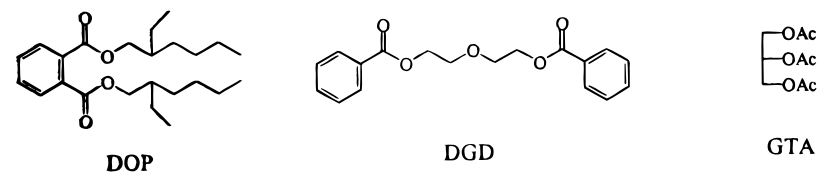

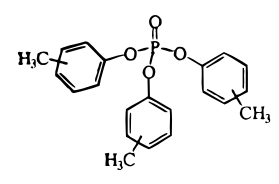

TCP

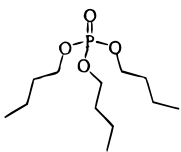

TBP
Figure 1. Plasticizers employed in this study (chlorinated paraffins omitted).

In our implementation of an artificial nose, arrays of carbon black/polymer composites comprised of 10-20 compositionally different detectors have been shown to differentiate efficiently between many organic vapors when assessed under controlled conditions in the laboratory. Swelling of the polymers leads to an increase in the dc resistance of the composite film, and the pattern of resistances is read though simple electronics and transferred to a processor for analysis. Distinctive patterns have allowed pairwise differentiation between species that differ in structure and polarity as well as between members of homologous series of, for example, alcohols or alkanes. ${ }^{14}$ Furthermore, the response of the carbon black/polymer composite sensors is linearly related to the concentration of a given vapor. ${ }^{12,14,15}$ However, even though hundreds of commercial polymers are potentially available for use, a major distinction between the existing conducting polymer composite electronic nose system and the mammalian olfactory system lies in sensor diversity. It would be useful if such diversity could be obtained from combining a limited number of feedstocks in a combinatorial fashion, as opposed to having to use different feed solutions for the fabrication of each detector. We report herein two implementations of a combinatorially based strategy to yield conducting polymer composite arrays having detectors with diverse vapor response properties.

\section{Methods}

Poly(methyl methacrylate), poly(vinyl chloride), poly(vinyl acetate), and polystyrene were used as received (Scientific Polymer Products, Inc.). The plasticizers di(2ethylhexyl)phthalate (DOP), diethylene glycol dibenzoate (DGD), glycerol triacetate (GTA), tributyl phosphate (TBP), chloroparaffin $(50 \% \mathrm{Cl}, \mathrm{CP} 50)$, chloroparaffin $(70 \% \mathrm{Cl}$, CP70), and tricresyl phosphate (TCP) (Figure 1) were used as received (Scientific Polymer Products, Inc.).

Vapor detectors were fabricated from solutions consisting of $20 \%$ carbon black (Black Pearls 2000, a furnace material produced by Cabot Co.), $20 \%$ plasticizer, and $60 \%$ polymer (by weight), or from solutions of $20 \%$ carbon black and $80 \%$ polymer, using methods described previously. ${ }^{14}$ The detector films were spray coated onto the surface of a glass slide onto 
which Au lines had been deposited by thermal evaporation. The changes in dc electrical resistance between the $\mathrm{Au}$ contact lines produced by these composites upon sorption of an analyte were monitored using a Keithley model 2002 meter connected to a Keithley model 7001 multiplexer. The flow system used to produce controlled concentrations of analyte and the associated electronics used to control the analyte delivery and data acquisition functions have been described previously. ${ }^{12}$ Resistance measurements of the composites were obtained before, during, and after exposure to controlled concentrations of hexane, toluene, chloroform, tetrahydrofuran, acetone, ethyl acetate, ethanol, or methanol. All exposures were adjusted to deliver the analyte at a concentration that was equal to $5 \%$ of the analyte's vapor pressure at room temperature. This produced analyte concentrations of (in units of parts per thousand (ppth)): methanol (6.63), ethanol (3.21), ethyl acetate (4.87), acetone (12.9), tetrahydrofuran (9.02), chloroform (10.7), and hexane (9.74).

The average steady-state dc resistance response of the composite was used to determine the differential resistance change of the detector, $\Delta R_{\max }$, in the presence of an analyte. Dividing this value by the baseline resistance, $R_{\mathrm{b}}$, yielded the relative differential resistance, $\Delta R_{\max } / R_{\mathrm{b}}$, which was the key quantity used to determine the response of a given detector to an analyte. Data were collected for all detectors exposed 15 times to each analyte, with each exposure being performed for $5 \mathrm{~min}$. Individual exposures to any given analyte were performed in random order during the data collection period.

The ability of each detector array to distinguish between different analytes was evaluated using the Fisher linear discriminant algorithm, which searches for the projection vector that maximizes the pairwise resolution factor for each set of analytes. The numerical metric produced by this method describes the separation of the data clusters when projected along this optimal discriminant vector. ${ }^{14}$ Resolution factors obtained from this process represent the statistical confidence of being able to identify correctly pairwise one of the analytes in the test set of interest from another, with resolution factors of $1.0,2.0$, and 3.0 corresponding to approximately 76,92 , and $98 \%$ confidence, respectively, of correctly identifying an analyte of a given solvent pair during a single subsequent exposure to the detector array.

\section{Results and Discussion}

Two approaches to obtaining sensor diversity from a limited number of chemical feedstocks have been explored in this work. One method uses a series of block copolymers synthesized from combinations of monomer feedstocks, and the other method involves modification of the properties of a base polymer composite vapor detector through addition of a series of plasticizers. ${ }^{16}$ In each case, a question of concern is whether detectors that contain the more compositionally-complex films have unique sorption properties for at least some members of a test set of analyte vapors. ${ }^{17}$

Figure 2 illustrates the effect that addition of various plasticizers had on the response of a carbon black/poly(vinyl acetate) composite vapor detector. As shown in the figure,

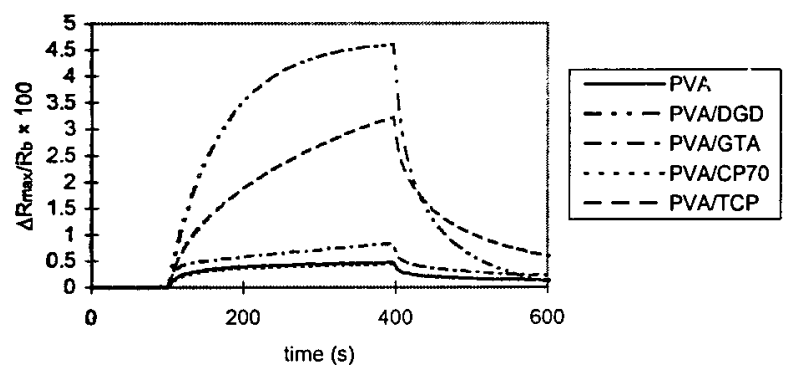

Figure 2. Response curves for a poly(vinyl acetate) (PVA)/carbon black composite detector compared to the response of plasticized poly(vinyl acetate)/carbon black detectors. In each case, the plasticizer indicated in the legend was present at $20 \mathrm{wt} \%$ in the solution used to form the detectors. The analyte, at $5 \%$ of its vapor pressure at room temperature (i.e., a concentration of 12.9 ppth of acetone in air), was exposed to the sensors starting on the abscissa at $100 \mathrm{~s}$ and ending at $400 \mathrm{~s}$. The $\Delta R_{\max } / R_{\mathrm{b}}$ data of the ordinate, where $R_{\mathrm{b}}$ is the drift-corrected baseline resistance of the detector prior to the exposure and $\Delta R_{\max }$ is the maximum differential resistance change upon exposure to the analyte relative to this baseline resistance value, clearly indicate that the plasticizer had a distinct effect on the response properties of the base polymer used in the carbon black/polymer composite detectors.

a relatively small $\Delta R_{\max } / R_{\mathrm{b}}$ response of the detector to 12.9 ppth of acetone was observed in the absence of an additive. In contrast, each plasticizer, despite being present in an equal mass fraction, resulted in a different response from the detector, with some of the responses being much larger than the responses of the unplasticized polymer composite film.

These differences in response permitted formation of a detector array that could distinguish between various analytes based on their differing array response patterns, even though only one base polymer was used in the formation of the detector array. The array that was evaluated consisted of different carbon black composite detectors, one formed using the unplasticized polymer and the other detectors formed from the same polymer through addition of a fixed, constant weight fraction of different plasticizers. Tables 1 and 2 present the resolution factors of such four- or five-detector arrays in differentiating pairwise between seven test analyte vapors, with the data in Table 1 for a poly(vinyl chloride)based array and the data in Table 2 for a poly(vinyl acetate)based array. Given that an of of 3 corresponds to $98 \%$ confidence of correctly differentiating between a pair of analytes on a single exposure to the detector array, both arrays formed from the base polymer with a series of plasticizers were clearly able to differentiate between the test analytes with high levels of confidence. When fewer detector elements (i.e., fewer different types of plasticizers) were employed, the observed resolution factors generally decreased for the test set of analytes under consideration (see Supporting Information). In practice, columns of such base polymers would be deposited onto the pixels of a substrate having active electronics and electrical contacts to the polymer films arranged into a rectangular array of unit cells. Different plasticizers would then be deposited along the rows of this substrate. Through use of a binary mixing process, a target of 1000 compositionally different detectors could thus be achieved through use of 30 different polymers with 30 different, compatible plasticizers. Similarly, as few as 30 total polymer and plasticizer feedstocks would be needed if a 
Table 1. Resolution Factors for a Four-Detector Array Consisting of Carbon Black Composites ${ }^{a}$

\begin{tabular}{|c|c|c|c|c|c|c|c|}
\hline & toluene & chloroform & THF & acetone & ethyl acetate & ethanol & methanol \\
\hline hexane & 7.4 & 12.7 & 13.3 & 22.0 & 14.7 & 10.2 & 24.0 \\
\hline toluene & & 6.1 & 9.7 & 12.5 & 9.4 & 10.4 & 15.7 \\
\hline chloroform & & & 4.5 & 8.3 & 6.1 & 9.7 & 19.2 \\
\hline THF & & & & 10.8 & 4.3 & 11.1 & 18.4 \\
\hline acetone & & & & & 10.9 & 16.9 & 24.0 \\
\hline ethyl acetate & & & & & & 12.4 & 22.5 \\
\hline ethanol & & & & & & & 20.0 \\
\hline
\end{tabular}

${ }^{a}$ One detector was formed from a composite of pure poly(vinyl chloride), and three other detectors were formed from composites of poly(vinyl chloride) that had been plasticized with DOP, DGD, or TCP, respectively. Analytes were presented to the detectors at a concentration of 5\% of their vapor pressure at room temperature in a background air ambient. A resolution factor of 3 represents a $98 \%$ statistical confidence for correct identification of one analyte of the chosen solvent pair relative to the other analyte in a single subsequent exposure to the detector array.

Table 2. Resolution Factors for a Five-Detector Array of Carbon Black Composites ${ }^{a}$

\begin{tabular}{|c|c|c|c|c|c|c|c|}
\hline & toluene & chloroform & THF & acetone & ethyl acetate & ethanol & methanol \\
\hline hexane & 11.0 & 23.7 & 14.4 & 60.8 & 13.2 & 21.1 & 23.9 \\
\hline toluene & & 17.8 & 9.0 & 35.5 & 9.4 & 12.3 & 15.9 \\
\hline chloroform & & & 9.0 & 8.6 & 7.7 & 9.4 & 10.8 \\
\hline $\mathrm{THF}$ & & & & 13.6 & 1.8 & 7.5 & 11.9 \\
\hline acetone & & & & & 11.6 & 32.8 & 40.9 \\
\hline ethyl acetate & & & & & & 8.1 & 12.7 \\
\hline ethanol & & & & & & & 6.5 \\
\hline
\end{tabular}

${ }^{a}$ One detector was formed from pure poly(vinyl acetate) as the polymer; the other four detectors were formed from composites of poly(vinyl acetate) that had been plasticized with DGD, GTA, CP70, or TCP, respectively. Analytes were presented to the detectors at 5\% of their vapor pressure at room temperature in a background air ambient. A resolution factor of 3 represents a $98 \%$ statistical confidence for correct identification of one analyte of the chosen solvent pair relative to the other analyte in a single subsequent exposure to the detector array.

ternary mixing process (and no compositional gradients) were used to fabricate the 1000 chemically different detector films.

A second method for potentially producing diversity in conducting polymer composites involves the use of block copolymers. Unlike polymer blends, block copolymers can be made from chemically diverse monomers without obtaining macrophase separation of the components. A potential drawback is the possibility that the response of the block copolymers could be a linear combination of the two homopolymers, thus yielding no unique sorption data that could be used to identify analytes. ${ }^{18}$

Block copolymers were synthesized using the living polymerization process of ring-opening metathesis polymerization, as depicted in Scheme $1 .{ }^{19}$ The monomers were chosen such that one monomer was very polar and the other monomer was relatively nonpolar, to maximize the difference in the sorption coefficient of the pure homopolymers in the presence of a range of analytes.

Figure 3 compares the response properties of carbon black composites of homopolymers formed from the two different monomers to the response properties of carbon black composite detectors formed from the block copolymerization reactions of these same two monomer units. Five different block copolymer compositions were evaluated during this experiment, and each analyte was presented to the resulting carbon black composite chemiresistors at $5 \%$ of the analyte's vapor pressure. The trend in the responses to hexane corresponds closely to what would be anticipated based on polarity of the polymers. Thus, the silyl-containing homopolymer exhibited the largest $\Delta R_{\max } / R_{\mathrm{b}}$ response. Also, the magnitude of the response decreased nearly linearly with increasing proportion of the polar block (Figure 3a). Similarly, methanol effectively swelled the polymers containing
Scheme 1. Synthesis of Block Copolymers for Sorption Studies $^{a}$
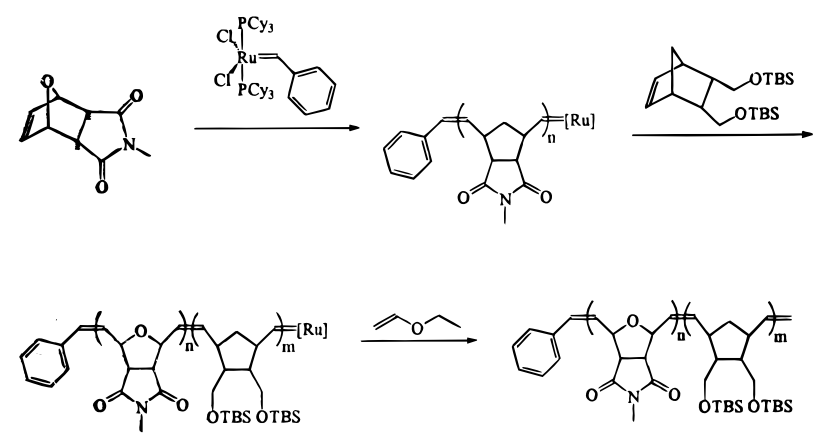

$a$ The first monomer formed the polar block, while the silyl-containing, second monomer formed the nonpolar block of the material.

the polar units, but the response decreased for polymers containing more than $50 \%$ of the silyl monomer (Figure $3 \mathrm{~b}$ ). Exposure to acetone, however, provided an example of an analyte in which the 50:50 block composition was the most responsive (Figure 3c). Different behavior was observed upon exposure to ethyl acetate (Figure 3d). These results demonstrate that the responses of block copolymers are not a simple function of their constitution and suggest that multiblock structures synthesized by living polymerization processes may offer an interesting approach to constructing large libraries of chemically diverse polymeric sensors.

The combination of the use of block copolymers and plasticizers suggests that a highly chemically diverse "nosechip" detector array could be formed from a limited number of feedstock solutions, if these feedstocks were arrayed spatially across a substrate to form compositionally distinct materials at each position in the array. Such a procedure would avoid the requirement of delivering a different 
(a)

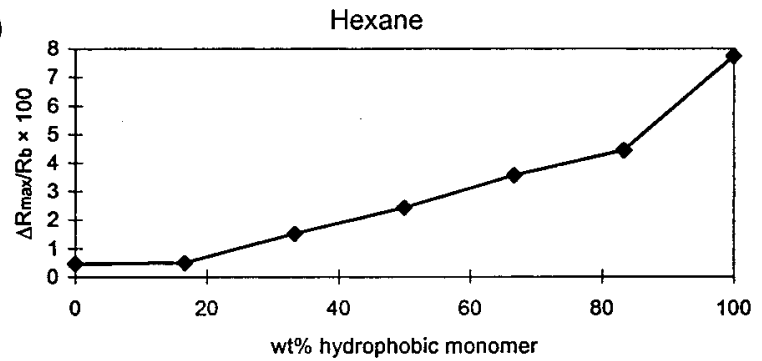

(b)

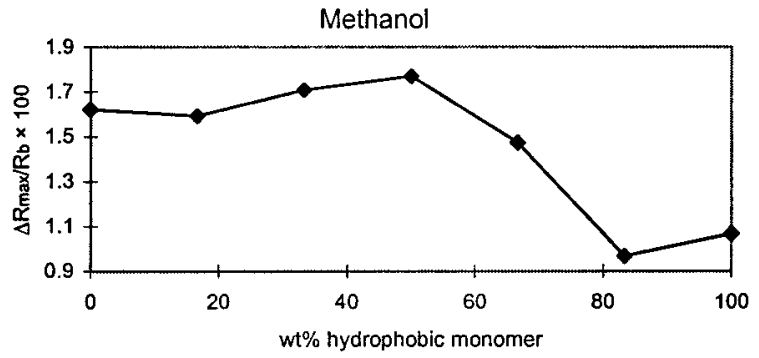

(c)

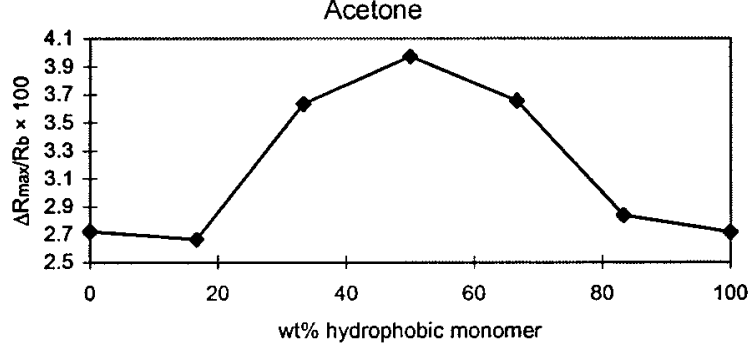

(d)

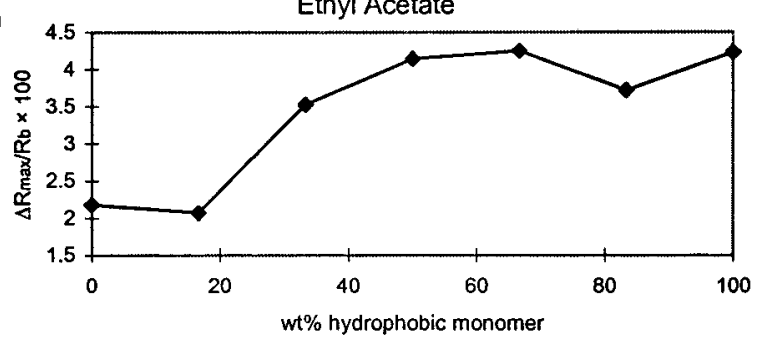

Figure 3. Response data for block copolymers as a function of polymer composition. The blocks were formed from living polymerization of a polar monomer (left) and a nonpolar (silyl-containing) monomer, as displayed in Scheme 1. Each analyte was present at $5 \%$ of its vapor pressure for a period of $300 \mathrm{~s}$, with the background gas being laboratory air. The values on the ordinate are the median maximum relative differential resistance responses, $\Delta R_{\max } / R_{\mathrm{b}}$, of each detector during the analyte exposure period. Data were obtained from 15 exposures to each analyte, with every individual analyte exposure performed in random order.

polymer from a different solution to each pixel. The electronics that form the basis for such a "nose-chip", having thousands of individually addressable pixels, are readily fabricated using conventional CMOS technology, and the behavior of such combinatorially-formed "nose-chips" is under investigation.

\section{Conclusions}

We have demonstrated a simple technique for altering the response properties of polymer-based chemiresistor vapor detectors through use of plasticizers. The approach should be applicable to a variety of implementations of electronic noses. In addition, a series of well-defined block copolymers was found to have sorption behavior that was not a linear combination of the properties of the two separate blocks. Taken together, these methods suggest approaches for forming large numbers of compositionally different detectors from a limited number of feedstock solutions.

Acknowledgment. This work was supported by DARPA, the Army Research Office, the Department of Energy, and NASA.

Supporting Information Available. Experimental details, resolution factors utilizing arrays of different numbers of detectors, a full description of resolution factors, and a representative table of $\Delta R_{\max } / R_{\mathrm{b}}$ values are available free of charge via the Internet at http://pubs.acs.org.

\section{References and Notes}

(1) Dickinson, T. A.; White, J.; Kauer, J. S.; Walt, D. R. Trends Biotechnol. 1998, 16, 250-258.

(2) Bartlett, P. N.; Elliott, J. M.; Gardner, J. W. Ann. Chim. 1997, 87, 33-44.

(3) Schaller, E.; Bosset, J. O.; Escher, F. Food Sci. Technol.-Lebensm.Wiss. Technol. 1998, 31, 305-316.

(4) Harsányi, G. Polymer films in sensor applications: technology, materials, devices and their characteristics; Technomic Pub. Co. Lancaster, 1995.

(5) Chang, S. M.; Iwasaki, Y.; Suzuki, M.; Tamiya, E.; Karube, I.; Muramatsu, H. Anal. Chim. Acta 1991, 249, 323-329.

(6) Grate, J. W.; Martin, S. J.; White, R. M. Anal. Chem. 1993, 65, A987-A996

(7) Grate, J. W.; Martin, S. J.; White, R. M. Anal. Chem. 1993, 65, A940-A948.

(8) Dickinson, T. A.; White, J.; Kauer, J. S.; Walt, D. R. Nature 1996, 382, 697-700.

(9) White, J.; Kauer, J. S.; Dickinson, T. A.; Walt, D. R. Anal. Chem. 1996, 68, 2191-2202.

(10) Krech, J. H.; RosePehrsson, S. L. Anal. Chim. Acta 1997, 341, 5362.

(11) Persaud, K. C.; Khaffaf, S. M.; Payne, J. S.; Pisanelli, A. M.; Lee, D. H.; Byun, H. G. Sens. Actuator B-Chem. 1996, 36, 267-273.

(12) Lonergan, M. C.; Severin, E. J.; Doleman, B. J.; Beaber, S. A.; Grubbs, R. H.; Lewis, N. S. Chem. Mater. 1996, 8, 2298-2312.

(13) Malnic, B.; Hirono, J.; Sato, T.; Buck, L. B. Cell 1999, 96, 713723.

(14) Doleman, B. J.; Lonergan, M. C.; Severin, E. J.; Vaid, T. P.; Lewis, N. S. Anal. Chem. 1998, 70, 4177-4190.

(15) Severin, E. J.; Doleman, B. J.; Lewis, N. S. Anal. Chem. 2000, 72, $658-668$.

(16) Plasticizers have been employed in polymeric electrolytes, and were found to have only a secondary effect on selectivity: Buhlmann, K.; Schlatt, B.; Cammann, K.; Shulga, A. Sens. Actuator B-Chem. 1998, 49, 156-165.

(17) A combinatorial library of dye impregnated vapor detectors has been fabricated by addition polymerization: Dickinson, T. A.; Walt, D. R.; White, J.; Kauer, J. S. Anal. Chem. 1997, 69, 3413-3418.

(18) Doleman, B. J.; Sanner, R. D.; Severin, E. J.; Grubbs, R. H.; Lewis, N. S. Anal. Chem. 1998, 70, 2560-2564.

(19) Weck, M.; Schwab, P.; Grubbs, R. H. Macromolecules 1996, 29, 1789-1793.

CC990056T 